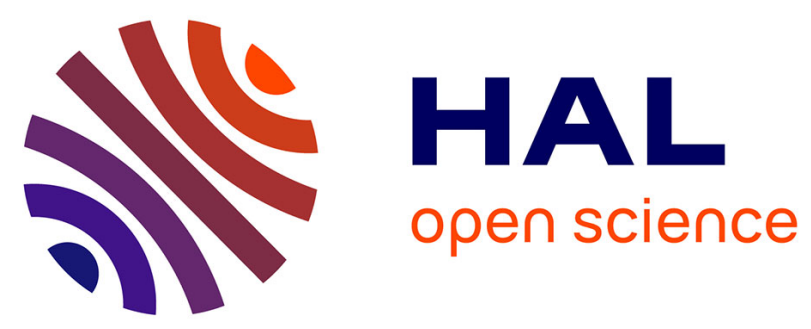

\title{
STOCHASTIC COMPARISONS AND AGING PROPERTIES OF AN EXTENDED GAMMA PROCESS
}

\author{
Zeina Al Masry, Sophie Mercier, Ghislain Verdier
}

\section{- To cite this version:}

Zeina Al Masry, Sophie Mercier, Ghislain Verdier. STOCHASTIC COMPARISONS AND AGING PROPERTIES OF AN EXTENDED GAMMA PROCESS. Journal of Applied Probability, 2021, 58 (1), pp.140-163. 10.1017/jpr.2020.74 . hal-02894591

\section{HAL Id: hal-02894591 \\ https://hal.science/hal-02894591}

Submitted on 9 Jul 2020

HAL is a multi-disciplinary open access archive for the deposit and dissemination of scientific research documents, whether they are published or not. The documents may come from teaching and research institutions in France or abroad, or from public or private research centers.
L'archive ouverte pluridisciplinaire HAL, est destinée au dépôt et à la diffusion de documents scientifiques de niveau recherche, publiés ou non, émanant des établissements d'enseignement et de recherche français ou étrangers, des laboratoires publics ou privés. 


\title{
STOCHASTIC COMPARISONS AND AGING PROPERTIES OF AN EXTENDED GAMMA PROCESS
}

ZEINA AL MASRY, ${ }^{*}$ FEMTO-ST, Univ. Bourgogne Franche-Comté, CNRS, ENSMM

SOPHIE MERCIER \& GHISLAIN VERDIER, ${ }^{* *}$ Universite de Pau et des Pays de l'Adour, E2S UPPA, CNRS, LMAP, Pau, France

\begin{abstract}
Extended gamma processes have been seen to be a flexible extension of standard gamma processes in the recent reliability literature, for cumulative deterioration modeling purpose. The probabilistic properties of the standard gamma process have been well explored since the 1970's, whereas those of its extension remain largely unexplored. In particular, stochastic comparisons between degradation levels modeled by standard gamma processes and aging properties for the corresponding level-crossing times are nowadays well understood. The aim of this paper is to explore similar properties for extended gamma processes and see which ones can be widen to this new context. As a by-product, new stochastic comparisons for convolutions of gamma random variables are also obtained.
\end{abstract}

Keywords: Reliability theory; Cumulative deterioration; Standard gamma process; Extended gamma process; Stochastic order; Aging properties

2010 Mathematics Subject Classification: Primary 60K10

Secondary 60E15;60G51

\section{Introduction}

Safety and dependability are crucial issues for many industrial systems, which have led to the development of a huge literature devoted to reliability theory. We are here concerned with industrial systems which deteriorate over time, and such that their degradation level can be synthesized through a univariate indicator. For this type of system, a common way is to model the evolution of its deterioration level over time through a univariate continuous-time stochastic process $\left(Y_{t}\right)_{t \geq 0}$, where $Y_{t}$ represents the degree of degradation at time $t$ (as measured by the univariate indicator). In such a setting, the system is often considered as failed as soon as its degradation level exceeds a predetermined failure threshold $\ell>0$. The time at which the failure occurs hence is

$$
\sigma_{\ell}=\inf \left\{t>0: Y_{t}>\ell\right\} \text {. }
$$

Most of the time, the degradation level of an industrial system is increasing over time, or at least has an increasing trend. Based on that, many different models have been envisioned in the literature such as Wiener process with positive drift (see, e.g., [7] and [8]), cumulative shock model (see, e.g., [23]), inverse Gaussian process (see, e.g., $[25]$ ) or inverse gamma processes (see, e.g., [10]). However, one of the most popular

\footnotetext{
* Postal address: Besançon cedex, 25000, France: zeina.almasry@femto-st.fr

** Postal address: Pau, 64000, France: sophie.mercier@univ-pau.fr; ghislain.verdier@univ-pau.fr
} 
model for cumulative degradation is the Standard Gamma Process (SGP), see [24] for a comprehensive overview of applications of the gamma process to reliability theory. Even in its non homogeneous version, a SGP has independent and non-negative (gamma distributed) increments and it is a non-homogeneous Lévy process (also called additive process by [19]). As any Lévy (or additive) process, a major drawback of a SGP is the fact that its variance-to-mean ratio remains constant over time, which can be quite restrictive for some applications. This has been highlighted by Guida et al. [11], who have provided a real data set of sliding wear data of four metal alloy specimens, where there is some "empirical evidence that the variance-to-mean ratio is not a constant but varies with [time]". To overcome this restriction, an extended version of the gamma process has been introduced by [4] and [9], which does not suffer from the same limitation. Whereas a standard non homogeneous gamma process is characterized by a shape function and a scale parameter, an Extended Gamma Process (EGP) has both a shape function and a scale function, as its scale parameter can evolve over time. The modeling flexibility of an EGP has been highlighted in [1], where it is shown that beyond the fact that the variance-to-mean ratio needs not remain constant over time as for an SGP, it is possible to model very different behaviors with an EGP: it can have trajectories mostly similar to those of an SGP, but it can also present exploding trajectories at a finite time or stabilizing trajectories, according to its parameters, and one single EGP can even have one part of its trajectories which are exploding and another part which are stabilizing, please see Proposition 1 and Example 1 in [1] for more details. Extended Gamma Processes hence are much more flexible than their standard version with respect to modeling purpose. This however has a cost and they are much less tractable from a mathematical point of view. Indeed, the probability density function of an EGP is not available in full form in a general setting. Also, there are no exact simulation procedures for its trajectories. This has lead Guida et al. [11] to consider a discrete-time version of an EGP, which can be viewed as a time discretization of a continuous-time EGP and is much easier to deal with. The authors however do not make the connection any further with continuous-time EGPs and the paper only deals with the discrete-time EGP. The idea of discretizing has been explored in details in [1], where the authors construct a discrete-time approximation of a general continuous-time EGP through discretization of the scale function of the general EGP. Some approximate simulation procedures are derived together with numerical tools for the approximate assessment of the distribution of a general EGP. The convergence of both approximate simulation procedures and numerical scheme for the assessment of the distribution is shown when the discretization step tends to zero. These tools make it possible to simulate trajectories and make numerical computation for an EGP.

For practical use, the statistical inference of a stochastic model also is an important task. As for EGPs, a first estimation procedure has been developed by [2] in a parametric context and for a specific observation scheme. It is based on a generalized method of moments, which relies on the full form expression available for the Laplace transform of an EGP (which is not the case of its probability density function). That was a first attempt but clearly, the subject deserves further studies based on e.g. different observation schemes.

Except from these recent papers, we are not aware of any other study of the probabilistic or statistical properties of an extended gamma process. The aim of this paper is to try to go a little further in the comprehension of such properties, focusing 
on stochastic comparisons and related properties in reliability theory.

Stochastic ordering and related results have been the subject of a huge literature with application in many different areas such as insurance, risk theory and finance (see [17] and [5]), queuing theory (see [22]) and in reliability theory, see [21] and [14], among others. In the context of a system with cumulative degradation modeled by a stochastic process, stochastic comparisons between two processes belonging to the same family (here EGPs) but with different parameters allow a better understanding of the influence of the parameters on the behavior of the corresponding system for instance. Related properties of interest in reliability concern the aging properties of failure times (as defined by (1)), which are of major interest for an adequate planning of preventive maintenance actions. As for SGPs, many results of stochastic comparisons and aging properties have already been obtained in the previous literature or can easily be derived from well-known properties of gamma distributions (see, e.g., [18] and [12]). In the following, we begin with a review of these published results, for sake of completeness and a better understanding of the new results for EGPs. Note also that the distribution of $Y_{t}$ in an EGP $\left(Y_{t}\right)_{t \geq 0}$ can be seen as an infinite convolution of heterogeneous gamma distributions with different rate parameters (details further). As will be seen, this leads us to develop new results for convolution of gamma distributions in order to derive some properties of EGPs.

The remainder of this paper is organized as follows. Section 2 provides some preliminaries on stochastic orders and related aging notions, together with some reminders of classical results for gamma distributions. Section 3 is devoted to stochastic comparisons and aging properties for a SGP and its related failure time, which are either well-known or direct consequences of Section 2. Similar properties are explored in Section 4, in the case of an extended gamma process. A summary of all the results for both SGP and EGP is finally provided in Section 5, for comparison purpose and a better understanding of the similarities and the differences between the results for the two processes, as well as some perspectives.

\section{Preliminaries}

In this section, we first recall the definition of the most common stochastic orders and aging notions. We refer to [21] for more details. Some existing results for the gamma distribution are then reminded.

Throughout the paper, "increasing", "decreasing" and "positive" mean "nondecreasing", "non-increasing" and "non negative", respectively.

\subsection{Stochastic orders}

Definition 1. Let $X$ (respectively $Y$ ) be a positive random variable with cumulative distribution function (cdf) $F_{X}$, survival function $\bar{F}_{X}$ and probability density function (pdf) $f_{X}$ (respectively $F_{Y}, \bar{F}_{Y}$ and $f_{Y}$ ). Then:

(a) $X$ is said to be smaller than $Y$ in the usual stochastic order (denoted by $X \prec$ sto $Y)$ if $\bar{F}_{X}(x) \leq \bar{F}_{Y}(x)$, for all $x \in \mathbb{R}$;

(b) $X$ is said to be smaller than $Y$ in the hazard rate order (denoted by $X \prec_{h r} Y$ ) if $\bar{F}_{Y}(t) / \bar{F}_{X}(t)$ is increasing in $t$ (on the union of the supports of $\bar{F}_{Y}$ and $\bar{F}_{X}$ );

(c) $X$ is said to be smaller than $Y$ in the reversed hazard rate order (denoted by $X \prec_{r h} Y$ ) if $F_{Y}(t) / F_{X}(t)$ is increasing in $t$ (on the union of the supports of $F_{Y}$ 
and $\left.F_{X}\right)$;

(d) $X$ is said to be smaller than $Y$ in the likelihood ratio order (denoted by $X \prec_{l r} Y$ ) if $f_{Y}(t) / f_{X}(t)$ is increasing in $t$ (on the union of the supports of $f_{Y}$ and $f_{X}$ ).

The likelihood ratio order implies both hazard rate and reversed hazard rate orders. Also, both of the latter imply the usual stochastic order.

Note that the stochastic order is equivalent to $\mathbb{E}(\varphi(X)) \leq \mathbb{E}(\varphi(Y))$ for all increasing function $\varphi$ for which the expectations exist. In addition, the definitions of the hazard rate, reversed hazard rate and likelihood ratio orders can be rewritten in a more common form for the reliability community:

Lemma 1. Let $X$ and $Y$ be two random variables. Then:

(a) $X \prec_{h r} Y$ if and only if $[X \mid X>l] \prec_{\text {sto }}[Y \mid Y>l]$ for all $l$;

(a) $X \prec_{r h} Y$ if and only if $[X \mid X \leq l] \prec_{\text {sto }}[Y \mid Y \leq l]$ for all $l$;

(a) $X \prec_{l r} Y$ if and only if $\left[X \mid l_{1} \leq X \leq l_{2}\right] \prec_{\text {sto }}\left[Y \mid l_{1} \leq Y \leq l_{2}\right]$ for all $l_{1} \leq l_{2}$.

The previous orders compare the locations of random variables. We now introduce orders that can be used for the joint comparison of location and dispersion.

Definition 2. Let $X$ and $Y$ be two positive random variables such that the support of $X$ is assumed to be included in the support of $Y$ and the support of $Y$ to be an interval. Then:

(a) $X$ is said to be smaller than $Y$ in the increasing convex order (denoted by $\left.X \prec_{i c x} Y\right)$ if $\mathbb{E}(\varphi(X)) \leq \mathbb{E}(\varphi(Y))$ for all increasing convex function $\varphi$.

(b) $X$ is said to be smaller than $Y$ in the increasing concave order (denoted by $\left.X \prec_{i c v} Y\right)$ if $\mathbb{E}(\varphi(X)) \leq \mathbb{E}(\varphi(Y))$ for all increasing concave function $\varphi$.

Provided the expectations exist, the monotone convex (respectively concave) order means that $\mathbb{E}(X) \leq \mathbb{E}(Y)$, plus the fact that $X$ is less (respectively more) variable than $Y$, in a stochastic sense (see [21, p.182]).

Note that the usual stochastic order implies both increasing convex and concave orders ([13]).

\subsection{Aging notions}

In this section, we provide the definitions and equivalent conditions of some aging properties.

Definition 3. Let $X$ be a positive random variable with cdf $F_{X}$, survival function $\bar{F}_{X}$ and pdf $f_{X}$ :

(a) $X$ is said to have the increasing failure rate (IFR) property if $\bar{F}_{X}$ is log-concave on $\mathbb{R}$;

(b) $X$ is said to have the decreasing failure rate (DFR) property if $\bar{F}_{X}$ is log-convex on $\mathbb{R}$;

(c) $X$ is said to have the decreasing reversed hazard rate (DRHR) property if $F_{X}$ is log-concave on $\mathbb{R}$. 
In the sequel, for the sake of brevity, we will say that $X$ is IFR (or DFR, or DRHR). Following [21, p.35 and p.42] and [20], the previous definitions are equivalent to the conditions below:

Lemma 2. Let $X$ be a random variable.

(a) $X$ is IFR if and only if $[X-t \mid X>t] \succ_{h r}\left[X-t^{\prime} \mid X>t^{\prime}\right]$ for all $t \leq t^{\prime}$;

(b) $X$ is DFR if and only if $[X-t \mid X>t] \prec_{h r}\left[X-t^{\prime} \mid X>t^{\prime}\right]$ for all $t \leq t^{\prime}$;

(c) $X$ is DRHR if and only if $[t-X \mid X \leq t] \prec_{h r}\left[t^{\prime}-X \mid X \leq t^{\prime}\right]$ for all $t \leq t^{\prime}$.

Remark 1. Note that the three points of the previous lemma remain valid if the usual stochastic order is used in place of the hazard rate order. If $X$ refers to the lifetime of a system, then $[X-t \mid X>t]$ represents the residual life of the system at time $t$ (given that the system still operates at time $t$ ). The IFR property for $X$ says that the residual life decreases when the system is aging, that is when $t$ increases. The interpretation for the DFR property is similar, with a reversed monotonicity. As for the DRHR property, imagine that the failure can only be observed through an inspection and that by an inspection at some time $t$, the system is observed to be failed. Then, $[t-X \mid X \leq t]$ represents the time since failure, which can be seen as an unavailability period. The DRHR property now means that the larger the inspection time, the longer the unavailability period, which can be seen as an aging property for $X$.

\subsection{Results for gamma distributed random variables}

We recall that the gamma distribution $\Gamma_{0}(a, b)$ with shape and scale parameters $a, b>0$, respectively, admits the following pdf:

$$
f(z)=\frac{b^{a}}{\Gamma(a)} z^{a-1} e^{-b z} \mathbb{1}_{\mathbb{R}_{+}}(z)
$$

with respect to Lebesgue measure, where $\Gamma(a)=\int_{0}^{\infty} s^{a-1} \exp (-s) d s$ for all $a>0$. The corresponding mean and variance are $a / b$ and $a / b^{2}$, respectively. Note also that if $Z_{1}, \cdots, Z_{n}$ are independent gamma distributed random variables with respective distributions $\Gamma_{0}\left(a_{1}, b\right), \cdots, \Gamma_{0}\left(a_{n}, b\right)$, where $a_{1}>0, \ldots, a_{n}>0, b>0$, then $b Z_{1}$ and $\sum_{i=1}^{n} Z_{i}$ are gamma distributed with parameters $\left(a_{1}, 1\right)$ and $\left(\sum_{i=1}^{n} a_{i}, b\right)$, respectively. Finally, we recall some classic results for gamma distributions:

Lemma 3. [16, p.62] Let $Z_{1}$ and $Z_{2}$ be gamma distributed random variables with parameters $\left(a_{1}, b_{1}\right)$ and $\left(a_{2}, b_{2}\right)$, respectively, where $a_{i}, b_{i}>0$ for $i=1,2$. Then:

(1) If $a_{1} \leq a_{2}$ and $b_{1} \geq b_{2}$, then $Z_{1} \prec_{l r} Z_{2}$, and hence $Z_{1} \prec_{h r} Z_{2}$ and $Z_{1} \prec_{r h} Z_{2}$;

(2) If $a_{1} \geq a_{2}$ and $a_{1} / b_{1} \leq a_{2} / b_{2}$, then $Z_{1} \prec$ icx $Z_{2}$;

(3) If $b_{1} \leq b_{2}$ and $a_{1} / b_{1} \leq a_{2} / b_{2}$ (and hence $a_{1} \leq a_{2}$ ), then $Z_{1} \prec_{i c v} Z_{2}$.

Lemma 4. [26, ] Let $Z_{i} \sim \Gamma_{0}\left(a_{i}, b_{i}\right), i=1, \ldots, n$, be independent gamma distributed random variables. Let us set $S_{Z}=\sum_{i=1}^{n} Z_{i}, U_{i}=b_{i} Z_{i} \sim \Gamma_{0}\left(a_{i}, 1\right)$ for $i \in\{1, \cdots, n\}$, $S_{U}=\sum_{i=1}^{n} U_{i} \sim \Gamma_{0}\left(\sum_{i=1}^{n} a_{i}, 1\right)$ and $a_{+}=\sum_{i=1}^{n} a_{i}$. Then,

- $\beta^{-1} S_{U} \prec_{a_{i} r} S_{Z}$ is equivalent to $\beta^{-1} S_{U} \prec_{\text {sto }} S_{Z}$ and it is true if and only if $\beta \geq \prod_{i=1}^{n} b_{i}^{\frac{a_{i}}{a_{+}}}$ 
- $\beta^{-1} S_{U} \prec_{l r} S_{Z}$ is equivalent to $\beta^{-1} S_{U} \prec_{r h} S_{Z}$ and it is true if and only if $\beta \geq \frac{1}{a_{+}} \sum_{i=1}^{n} a_{i} b_{i}$.

Lemma 5. [15, Proposition 9.A.3] Let $Z \sim \Gamma_{0}\left(a, b_{0}\right)$. Then $Z$ is IFR if $a \geq 1$, DFR if $a \leq 1$ and DRHR for all $a>0$.

\section{Standard Gamma Processes}

We here recall the definitions as well as some properties of SGPs. Throughout the paper, we let $A: \mathbb{R}_{+} \rightarrow \mathbb{R}_{+}$to be an increasing and continuous function with $A(0)=0$ and $a($.$) to be its derivative (which exists almost surely).$

\subsection{Definition}

Let $b_{0}$ be a positive constant. Recall that a standard (non-homogeneous) gamma process $\boldsymbol{Y}=\left(Y_{t}\right)_{t \geq 0} \sim \Gamma_{0}\left(A(\cdot), b_{0}\right)$, with $A(\cdot)$ as shape function and $b_{0}$ as scale parameter, is a stochastic process with independent, non-negative and gamma distributed increments:

$$
Y_{t}-Y_{s} \sim \Gamma_{0}\left(A(t)-A(s), b_{0}\right),
$$

for all $0<s<t$, and such that $Y_{0}=0$ a.s.. The pdf of an increment $Y_{t}-Y_{s}$ is then given by

$$
f(x)=\frac{b_{0}^{A(t)-A(s)}}{\Gamma(A(t)-A(s))} x^{A(t)-A(s)-1} \exp \left(-b_{0} x\right), \forall x \geq 0 .
$$

The mean and variance of $Y_{t}$ are given by $\mathbb{E}\left[Y_{t}\right]=A(t) / b_{0}$ and $\mathbb{V}\left[Y_{t}\right]=A(t) / b_{0}^{2}$ for all $t \geq 0$, respectively. Hence, the variance-to-mean ratio $\mathbb{V}\left[Y_{t}\right] / \mathbb{E}\left[Y_{t}\right]=b_{0}$ is constant over time.

\subsection{Stochastic ordering and aging properties for SGPs}

We first review aging properties and stochastic comparisons for the increments of a given SGP, and next provide stochastic comparisons between two SGPs with different parameters.

Based on Section 2.3 (and particularly on Lemmas 3 and 5), the three following lemmas are easily obtained.

Lemma 6. Let $\boldsymbol{Y}=\left(Y_{t}\right)_{t \geq 0}$ be a $S G P$ with shape function $A(\cdot)$ and scale parameter $b_{0}$. Then,

- if $A(t+h)-A(t) \geq 1, Y_{t+h}-Y_{t}$ is IFR; in particular $Y_{t}$ is IFR as soon as $A(t) \geq 1$

- if $A(t+h)-A(t) \leq 1, Y_{t+h}-Y_{t}$ is DFR; in particular $Y_{t}$ is DFR as soon as $A(t) \leq 1$

- $Y_{t+h}-Y_{t}$ and $Y_{t}$ are DRHR for all $t$ and all $h$.

Lemma 7. Let $\boldsymbol{Y}=\left(Y_{t}\right)_{t \geq 0}$ be a $S G P$ with shape function $A(\cdot)$ and scale parameter $b_{0}$. Then,

- if $0 \leq s \leq t$, then $Y_{s} \prec_{l r} Y_{t}$;

- if $A(\cdot)$ is convex, then $Y_{s+h}-Y_{s} \prec_{l r} Y_{t+h}-Y_{t}$ for all $0 \leq s \leq t$ and $h \geq 0$;

- if $A(\cdot)$ is concave, then $Y_{s+h}-Y_{s} \succ_{l r} Y_{t+h}-Y_{t}$ for all $0 \leq s \leq t$ and $h \geq 0$. 
Lemma 8. Let $\boldsymbol{Y}^{(i)}=\left(Y_{t}^{(i)}\right)_{t \geq 0} \sim \Gamma_{0}\left(A_{i}(\cdot), b_{i}\right)$ for $i \in\{1,2\}$.

- If $A_{1}(t) \leq A_{2}(t)$ and $b_{1} \geq b_{2}$, then $Y_{t}^{(1)} \prec_{l r} Y_{t}^{(2)}$;

- If $A_{2}(\cdot)-A_{1}(\cdot)$ is an increasing function and $b_{1} \geq b_{2}$, then for all $t, h \geq 0$, $Y_{t+h}^{(1)}-Y_{t}^{(1)} \prec_{l r} Y_{t+h}^{(2)}-Y_{t}^{(2)}$;

- If $A_{1}(t) \geq A_{2}(t)$ and $\frac{A_{1}(t)}{b_{1}} \leq \frac{A_{2}(t)}{b_{2}}$, then $Y_{t}^{(1)} \prec_{i c x} Y_{t}^{(2)}$;

- If $A_{2}(\cdot)-A_{1}(\cdot)$ is a decreasing function and $\frac{A_{2}(\cdot)}{b_{2}}-\frac{A_{1}(\cdot)}{b_{1}}$ is an increasing function, then $Y_{t+h}^{(1)}-Y_{t}^{(1)} \prec_{i c x} Y_{t+h}^{(2)}-Y_{t}^{(2)}$ for all $t, h \geq 0$;

- If $A_{1}(t) \leq A_{2}(t), \frac{A_{1}(t)}{b_{1}} \leq \frac{A_{2}(t)}{b_{2}}$ and $b_{1} \leq b_{2}$, then $Y_{t}^{(1)} \prec_{i c v} Y_{t}^{(2)}$;

- If $b_{1} \leq b_{2}$ and $\frac{A_{2}(\cdot)}{b_{2}}-\frac{A_{1}(\cdot)}{b_{1}}$ is an increasing function (and necessarily $A_{2}(\cdot)-A_{1}(\cdot)$ too), then $Y_{t+h}^{(1)}-Y_{t}^{(1)} \prec_{i c v} Y_{t+h}^{(2)}-Y_{t}^{(2)}$ for all $t, h \geq 0$.

Finally, we come to present the properties of the crossing time of a given threshold $\ell$, which can stand for the failure time of a system modeled by a SGP, as mentioned in the introduction. Note that in the context of a SGP which is a pure jump process, the level $\ell$ is almost surely crossed by a jump, so that the level $\ell$ is almost surely never hit. That is why we call it a crossing time, and not a hitting time, which is the common vocabulary for continuous processes such as Wiener processes for instance. The same remark is valid for an EGP hereafter. Note also that a threshold crossing time can also represent the time for a required preventive maintenance action, if $\ell$ stands for a preventive threshold (lower than the failure threshold). In that case, the maintenance action is triggered as soon as the deterioration level is beyond $\ell$ (at time $\sigma_{\ell}$ ), the aim being to avoid failures that could have unsafe or costly consequences.

The results below can be found in [18] and [12].

Lemma 9. Let $\boldsymbol{Y}=\left(Y_{t}\right)_{t \geq 0}$ be a $S G P$ with shape function $A(\cdot)$ and scale parameter $b_{0}$, and let $\ell>0$.

- If $A(\cdot)$ is convex, then $\sigma_{\ell}$ is IFR.

- If $A(\cdot)$ is concave, then $\sigma_{\ell}$ is DRHR.

\section{Extended gamma processes}

The aim of this section is to study how the results of Section 3 on SGPs can be adapted to EGPs. We first remind basic definitions and properties of EGPs in Subsection 4.1. Aging properties and comparison results of EGP increments are next discussed in Subsections 4.2 and 4.3. The comparison between two different EGPs is investigated in Subsection 4.4. Finally, aging properties of threshold crossing times are studied in the context of EGPs in Subsection 4.5.

In all the sequel, we refer to a SGP by $\boldsymbol{Y}=\left(Y_{t}\right)_{t \geq 0}$ and to an EGP by $\boldsymbol{X}=\left(X_{t}\right)_{t \geq 0}$.

\subsection{Definition}

Let $b: \mathbb{R}_{+}^{*} \rightarrow \mathbb{R}_{+}^{*}$ be a measurable positive function such that:

$$
\int_{(0, t]} \frac{a(s)}{b(s)} d s<\infty, \quad \forall t>0 .
$$


Following [4] and [9], the process $\boldsymbol{X}=\left(X_{t}\right)_{t \geq 0}$ is said to be an EGP with shape function $A(\cdot)$ and scale function $b(\cdot)$ if $X_{t}$ can be represented as a stochastic integral with respect to a SGP $\boldsymbol{Y}$ with $Y_{t} \sim \Gamma_{0}(A(t), 1)$ :

$$
X_{t}=\int_{(0, t]} \frac{d Y_{s}}{b(s)}, \quad \forall t>0,
$$

and $X_{0}=0$. If $b(\cdot)$ is constant and equal to $b_{0}$, the EGP simply reduces to a SGP $\Gamma_{0}\left(A(t), b_{0}\right)$. An EGP is much more flexible than a SGP and can be proved to have independent increments, and its distribution to be infinitely divisible. It allows to model many possible behaviors for the system deterioration (see [1] for more precision). Also, an explicit formula is available for the Laplace transform of an increment ([4]), with

$$
\mathcal{L}_{X_{t+h}-X_{t}}(\lambda):=\mathbb{E}\left(e^{-\lambda\left(X_{t+h}-X_{t}\right)}\right)=\exp \left(-\int_{t}^{t+h} \log \left(1+\frac{\lambda}{b(s)}\right) a(s) d s\right),
$$

for all $t, \lambda \geq 0$ and $h>0$.

Finally, the mean and variance of an EGP are given by

$$
\mathbb{E}\left(X_{t}\right)=\int_{(0, t]} \frac{a(s)}{b(s)} d s \text { and } \mathbb{V}\left(X_{t}\right)=\int_{(0, t]} \frac{a(s)}{b(s)^{2}} d s, \quad \forall t>0 .
$$

\subsection{Aging properties of EGP's increments}

We first explore the aging properties of an EGP increment, as provided by Lemma 6 in the case of a SGP. We start with the DRHR property. With that aim, we begin with a technical lemma.

Lemma 10. Let $Z_{i} \sim \Gamma_{0}\left(a_{i}, b_{i}\right), i=1, \ldots, n$, be $n$ independent gamma distributed random variables. Then $\sum_{i=1}^{n} Z_{i}$ is DRHR.

Proof. It is a direct consequence of the closure property of DRHR random variables under convolution [21, Corollary 1.B.63.], and of the fact that gamma random variables are DRHR (see Lemma 6).

Next proposition shows that the DRHR property of a SGP increment remains valid for an EGP.

Proposition 1. Let $\boldsymbol{X}=\left(X_{t}\right)_{t \geq 0} \sim \Gamma(A(\cdot), b(\cdot))$ be an EGP. Then $X_{t}$ and $X_{t+h}-X_{t}$ are DRHR for all $h \geq 0$ and all $t \geq 0$.

Proof. Note that it is enough to show that $X_{t+h}-X_{t}$ is DRHR. The proof is divided into two parts. Following some arguments developed in [1], we first assume that the scale function $b$ (or the rate function $1 / b$ ) is a piecewise constant function given by:

$$
\frac{1}{b(v)}=\sum_{i=0}^{m} \frac{1}{b_{i}} \mathbb{1}_{\left(u_{i}, u_{i+1}\right]}(v) \text { for all } v \geq 0
$$

where $u_{0}=0<u_{i}<u_{i+1}<u_{m+1} \leq+\infty$ for all $1 \leq i \leq m-1$ and $b_{i}>0$ for all $1 \leq i \leq m$. Assuming $u_{i_{t}}<t \leq u_{i_{t}+1}, u_{i_{t+h}}<t+h \leq u_{i_{t+h}+1}$, and without loss of 
generality that $u_{i_{t}+1}<u_{i_{t+h}}$ we have:

$$
\begin{aligned}
X_{t+h}-X_{t} & =\frac{1}{b_{i_{t}}} \int_{\left(t, u_{i_{t}+1}\right]} d Y_{v}+\sum_{i=i_{t}+1}^{i_{t+h}-1} \frac{1}{b_{i}} \int_{\left(u_{i}, u_{i+1}\right]} d Y_{v}+\frac{1}{b_{i_{t+h}}} \int_{\left(u_{i_{t+h}}, t+h\right]} d Y_{v} \\
& =\frac{1}{b_{i_{t}}}\left(Y_{u_{i_{t}+1}}-Y_{t}\right)+\sum_{i=i_{t}+1}^{i_{t+h}-1} \frac{1}{b_{i}}\left(Y_{u_{i+1}}-Y_{u_{i}}\right)+\frac{1}{b_{i_{t+h}}}\left(Y_{t+h}-Y_{u_{i_{t+h}}}\right)
\end{aligned}
$$

where

$$
\begin{aligned}
\frac{1}{b_{i_{t}}}\left(Y_{u_{i_{t}+1}}-Y_{t}\right) & \sim \Gamma_{0}\left(A\left(u_{i_{t}+1}\right)-A(t), b_{i_{t}}\right), \\
\frac{1}{b_{i}}\left(Y_{u_{i+1}}-Y_{u_{i}}\right) & \sim \Gamma_{0}\left(A\left(u_{i+1}\right)-A\left(u_{i}\right), b_{i}\right), \\
\text { and } \frac{1}{b_{i_{t+h}}}\left(Y_{t+h}-Y_{u_{i_{t+h}}}\right) & \sim \Gamma_{0}\left(A(t+h)-A\left(u_{i_{t+h}}\right), b_{i_{t+h}}\right) .
\end{aligned}
$$

The increment $X_{t+h}-X_{t}$ can therefore be written as a convolution of independent gamma random variables. This shows, by Lemma 10, that $X_{t+h}-X_{t}$ is DRHR when $b(\cdot)$ is piecewise constant.

We now consider a general function $b: \mathbb{R}_{+}^{*} \rightarrow \mathbb{R}_{+}^{*}$. Then, there exists an increasing sequence $\left(\beta^{(n)}(\cdot)\right)_{n \in \mathbb{N}}$ of piecewise constant functions of the shape (7) that converges to $\frac{1}{b}$. Let $\left(X_{t}^{(n)}\right)_{t \geq 0} \sim \Gamma\left(A(\cdot), b^{(n)}(\cdot)\right)$ with $b^{(n)}(t)=\frac{1}{\beta^{(n)}(t)}$. We have:

$$
\lim _{n \rightarrow+\infty} \mathcal{L}_{X_{t+h}^{(n)}-X_{t}^{(n)}}(\lambda)=\mathcal{L}_{X_{t+h}-X_{t}}(\lambda) \text { for all } \lambda>0
$$

by the monotone convergence theorem so that

$$
X_{t+h}^{(n)}-X_{t}^{(n)} \stackrel{\mathcal{D}}{\rightarrow} X_{t+h}-X_{t} \text { when } n \rightarrow+\infty
$$

for all $t>0$, where the symbol $\stackrel{\mathcal{D}}{\rightarrow}$ stands for convergence in distribution. The result is next easily derived from the piecewise constant case and letting $n \rightarrow+\infty$ (as the DRHR property is preserved by convergence in distribution, see [21]).

We now come to the DFR property of an EGP increment, in the case where $A(t+h)-A(t)$ is smaller than 1 . Here again, we begin with a technical lemma.

Lemma 11. Let $Z_{i} \sim \Gamma_{0}\left(a_{i}, b_{i}\right), i=1, \ldots, n$, be $n$ independent gamma distributed random variables such that $\sum_{i=1}^{n} a_{i} \leq 1$ (so that $a_{i} \leq 1$ for all $i \in\{1, \cdots, n\}$ ). Then $\sum_{i=1}^{n} Z_{i}$ is DFR.

Proof. The DFR property is known not to be stable through convolution in general, so that the proof of Lemma 10 for the DRHR case should be adapted. Let us set $S_{Z}=$ $\sum_{i=1}^{n} Z_{i}, U_{i}=b_{i} Z_{i} \sim \Gamma_{0}\left(a_{i}, 1\right)$ for $i \in\{1, \cdots, n\}$ and $S_{U}=\sum_{i=1}^{n} U_{i} \sim \Gamma_{0}\left(\sum_{i=1}^{n} a_{i}, 1\right)$.

Using a similar argument as in the proof of Theorem 3 of [26], we may write: 


$$
S_{Z}=\sum_{i=1}^{n} \frac{1}{b_{i}} U_{i}=V S_{U}
$$

with

$$
V=\sum_{i=1}^{n} \frac{1}{b_{i}} \frac{U_{i}}{S_{U}}
$$

The random vector $\left(\frac{U_{1}}{S_{U}}, \cdots, \frac{U_{n}}{S_{U}}\right)$ is known to be independent on $S_{U}$ so that $V$ also is independent on $S_{U}$. Then:

$$
f_{S_{Z}}(z)=\int_{\mathbb{R}_{+}} f_{V S_{U} \mid V=v}(z) f_{V}(v) d v
$$

with $f_{V S_{U} \mid V=v}$ the conditional pdf of $V S_{U}$ given that $V=v$. The distribution of $S_{Z}$ appears therefore as a mixture of the $v S_{U}$ 's. As $\sum_{i=1}^{n} a_{i} \leq 1$, we know that $S_{U}$ is DFR and $v S_{U}$ as well. Then, the distribution of $S_{Z}$ appears as a mixture of DFR distributions and since the DFR property is closed under mixture [15], the proof is completed.

Proposition 2. Let $\boldsymbol{X}=\left(X_{t}\right)_{t \geq 0} \sim \Gamma(A(\cdot), b(\cdot))$ be an EGP. Let $h \geq 0$ and $t \geq 0$ be such that $A(t+h)-A(t) \leq 1$. Then $X_{t+h}-X_{t}$ is DFR. In particular, $X_{t}$ is DFR as soon as $A(t) \leq 1$.

Proof. We can use similar arguments as for the proof of Proposition 1 as the DFR property is also preserved by convergence in distribution, see [21].

We now explore the possibly IFR property in the case where $A(t+h)-A(t)$ is larger than 1 , and for that, a numerical example is considered.

Example 1. Let us consider $A(t)=t$ (which is both a concave and convex function) and let $b(t)=10 \mathbb{1}_{[0,0.75)}(t)+0.1 \mathbb{1}_{[0.75, \infty)}(t)$ (decreasing function). The function $\log \left(\bar{F}_{X_{1}}\right)$ is plotted in Figure 1 for $t=1$ (and hence $A(t)=1$ ). As can be seen, $\log \left(\bar{F}_{X_{1}}\right)$ is not a concave function, which shows that $X_{1}$ is not IFR.

Considering the increasing function $b(t)=0.1 \mathbb{1}_{[0,0.25)}(t)+10 \mathbb{1}_{[0.25, \infty)}(t)$ provides the same distribution for $X_{1}$ (convolution of the gamma distributions $\Gamma_{0}(0.25,0.1)$ and $\left.\Gamma_{0}(0.75,10)\right)$ and the same conclusion.

Based on the previous example, we can see that even considering a convex or a concave shape function and a monotonous scale function, an increment $X_{t+h}-X_{t}$ needs not be IFR when the shape parameter $A(t+h)-A(t)$ is larger than 1 . Hence, it seems difficult to find sufficient conditions to ensure the IFR property of an increment. As a by-product, we observe that not all aging properties provided in Lemma 6 for SGPs remain true for an EGP.

\subsection{Monotonicity of an EGP and stochastic comparison between incre- ments}

We here explore how to adapt the results of Lemma 7 in the case of an EGP. We first look at the possible monotonicity of an EGP with respect to the likelihood ratio order. For that, a numerical example is considered. 


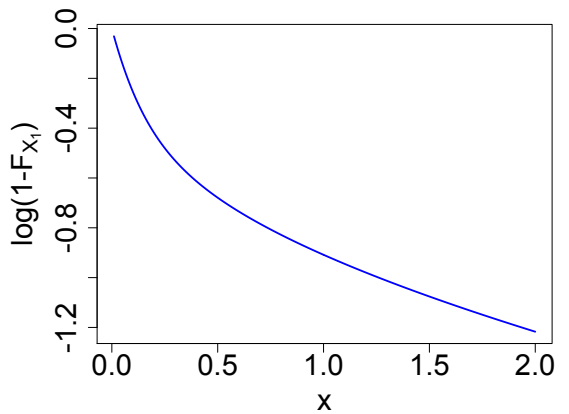

Figure 1: Plot of $\log \left(\bar{F}_{X_{1}}(t)\right)$ with respect to $t$ for $A(t)=t$ and $b(t)=10 \mathbb{1}_{[0,0.75)}(t)+$ $0.1 \mathbb{1}_{[0.75, \infty)}(t)$, Example 1 .

Example 2. Let us consider $A(t)=t$ and $b(t)=0.1 \mathbb{1}_{[0,0.25)}(t)+10 \mathbb{1}_{[0.25, \infty)}(t)$. The function $r(x)=\bar{F}_{X_{1}}(x) / \bar{F}_{X_{0.25}}(x)$ is plotted in Figure 2. We can observe that the function $r(x)$ is not monotonous with respect to $x$. Based on Definition 1, we can derive that $X_{0.25}$ and $X_{1}$ are not comparable with respect to the hazard rate order. Hence, they are not comparable either with respect to the likelihood ratio order.

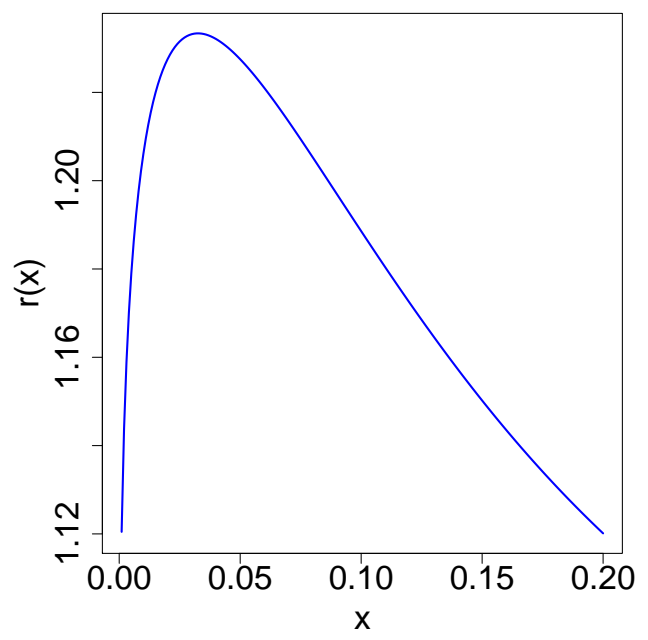

Figure 2: Plot of $r(x)=\bar{F}_{X_{1}}(x) / \bar{F}_{X_{0.25}}(x)$ as a function of $x$ for $A(t)=t$ and $b(t)=0.1 \mathbb{1}_{[0,0.25)}(t)+10 \mathbb{1}_{[0.25, \infty)}(t)$, Example 2.

Remark 2. Based on the previous example, we can see that the relationship $X_{s} \prec_{l r} X_{t}$ for $s<t$ is not valid in a general setting. To be more specific, considering a convex or a concave shape function, and an increasing scale function, the property needs not be true. However, we could not find any numerical counterexample with $A(t)=t$ (both convex and concave) and a decreasing scale function, and the property might hence be true under such assumptions. Unfortunately, we have not been able to prove it so that 
the question remains open. Note that the proof would require to get likelihood-ratio comparison results for convolution of heterogeneous gamma distributions with shape parameter smaller than 1 (tending to 0, in a similar spirit as for the proof of Proposition 1). This seems quite difficult as it seems that all the results from the literature are valid for shape parameters larger than 1 (see [27] for instance). It hence is quite a challenging (open) question.

Even though, under specific conditions, we were not able to conclude about the possible likelihood-ratio monotonicity of an EGP, we now show its monotonicity with respect to the reversed hazard rate order out of any conditions.

Proposition 3. Let $\boldsymbol{X}=\left(X_{t}\right)_{t \geq 0} \sim \Gamma(A(t), b(t))$ be an EGP. Then, for all $s<t$, we have

$$
X_{s} \prec_{r h} X_{t} \text {. }
$$

Proof. Let $s<t$. As $X_{t}-X_{s} \geq 0$, it is easy to check that

$$
0 \prec_{r h} X_{t}-X_{s} .
$$

Since $X_{s}$ is DRHR (see Proposition 1), we derive from [21, Lemma 1.B.44.] that $X_{s}=0+X_{s} \prec_{r h} X_{t}=\left(X_{t}-X_{s}\right)+X_{s}$, which achieves the proof.

We now provide comparison results between increments in the reversed hazard rate ordering. We begin with a technical lemma.

Lemma 12. Let $Z_{i}^{(1)} \sim \Gamma_{0}\left(a_{i}, b_{i}\right)$ and $Z_{i}^{(2)} \sim \Gamma_{0}\left(c_{i}, d_{i}\right), i=1, \ldots, n$, such that $a_{i} \leq c_{i}$ and $b_{i} \geq d_{i}$ for all $i$. Then,

$$
\sum_{i=1}^{n} Z_{i}^{(1)} \prec_{r h} \sum_{i=1}^{n} Z_{i}^{(2)}
$$

Proof. From Lemma 3, we have

$$
Z_{i}^{(1)} \prec_{l r} Z_{i}^{(2)} \forall i=1, \ldots, n
$$

so that

$$
Z_{i}^{(1)} \prec_{r h} Z_{i}^{(2)} \forall i=1, \ldots, n .
$$

As the reversed hazard rate order is stable under the convolution of DRHR random variables [21, Theorem 1.B.45], the result follows.

Proposition 4. Let $\boldsymbol{X}=\left(X_{t}\right)_{t \geq 0} \sim \Gamma(A(t), b(t))$ be an EGP. Assume that:

- $A(\cdot)$ is convex,

- $b(\cdot)$ is decreasing.

Then,

$$
X_{s+h}-X_{s} \prec_{r h} X_{t+h}-X_{t}
$$

for all $s \leq t$ and all $h>0$.

Proof. Let $s \leq t \leq t+h$. Here again, we first consider the case where $b(\cdot)$ is piecewise constant (and decreasing). Then, $(0, h]$ is the union of a finite number of disjoint intervals $\left(u_{i}, u_{i+1}\right], 0 \leq i \leq n$ such that $b(s+z)$ is constant for all $z \in\left(u_{i}, u_{i+1}\right]$ 
and all $0 \leq i \leq n$. Cutting each of these intervals into pieces when necessary, we can construct disjoint intervals $\left(v_{j}, v_{j+1}\right]$ for $0 \leq j \leq m$ with $v_{0}=0$ and $v_{m+1}=h$, such that $\cup_{j=0}^{m}\left(v_{j}, v_{j+1}\right]=(0, h]$ and on which both $b(s+z)$ and $b(t+z)$ are constant for all $z \in\left(v_{j}, v_{j+1}\right]$ and all $0 \leq j \leq m$. This provides:

$$
\begin{aligned}
& X_{t+h}-X_{t}=\sum_{j=0}^{m} \frac{1}{b\left(t+v_{j+1}\right)}\left(Y_{t+v_{j+1}}-Y_{t+v_{j}}\right)=\sum_{j=0}^{m} Z_{j}^{(t)}, \\
& X_{s+h}-X_{s}=\sum_{j=0}^{m} \frac{1}{b\left(s+v_{j+1}\right)}\left(Y_{s+v_{j+1}}-Y_{s+v_{j}}\right)=\sum_{j=0}^{m} Z_{j}^{(s)} .
\end{aligned}
$$

For all $j=1, \ldots, m$, we have $Z_{j}^{(z)} \sim \Gamma_{0}\left(A\left(z+v_{j+1}\right)-A\left(z+v_{j}\right), b\left(z+v_{j+1}\right)\right)$ for $z \in\{s, t\}$ with

$$
A\left(s+v_{j+1}\right)-A\left(s+v_{j}\right) \leq A\left(t+v_{j+1}\right)-A\left(t+v_{j}\right)
$$

and

$$
b\left(s+v_{j+1}\right) \geq b\left(t+v_{j+1}\right)
$$

because $A$ is convex and $b$ is decreasing. From Lemma 12, it then comes:

$$
X_{s+h}-X_{s} \prec_{r h} X_{t+h}-X_{t} .
$$

For a general decreasing function $b$, we proceed as in the proof of Proposition 1 and the result follows since $\prec_{r h}$ is preserved by convergence in distribution.

Reversing the assumptions, we obtain a reversed inequality, as provided in the following proposition. The proof is similar and it is omitted.

Proposition 5. Let $\boldsymbol{X}=\left(X_{t}\right)_{t \geq 0} \sim \Gamma(A(t), b(t))$ be an EGP. Assume that:

- $A(\cdot)$ is concave,

- $b(\cdot)$ is increasing.

Then,

$$
X_{s+h}-X_{s} \succ_{r h} X_{t+h}-X_{t}
$$

for all $s \leq t$ and all $h>0$.

Remark 3. In Propositions 4 and 5, the monotonicity of $b(\cdot)$ is essential. Indeed, it is not difficult to provide a counterexample to the previous results when the monotonicity assumption is not satisfied any more. Let $A(t)=t$ and $b(t)=1 / 2 \mathbb{1}_{[0,1)}(t)+1 / 3 \mathbb{1}_{[1,2)}(t)+$ $1 \mathbb{1}_{[2,3)}(t)+1 / 4 \mathbb{1}_{[3, \infty)}(t)$ (which is not monotonic). Then,

$$
X_{2}-X_{0} \stackrel{\mathcal{D}}{=} 2 Y_{1}+3 Y_{2} \text { and } X_{4}-X_{2} \stackrel{\mathcal{D}}{=} Y_{3}+4 Y_{4}
$$

where $\stackrel{\mathcal{D}}{=}$ means "is identically distributed as" and where $Y_{i} \sim \Gamma(1,1)$ for $i=1, \ldots, 4$. As noticed by [6], we have $V\left[X_{2}-X_{0}\right]<V\left[X_{4}-X_{2}\right]$, so that the distributions of the two increments are not identical. They however share the same expectation $\left(E\left[X_{2}-X_{0}\right]=E\left[X_{4}-X_{2}\right]\right)$, which entails that the sign of $F_{X_{2}-X_{0}}(x)-F_{X_{4}-X_{2}}(x)$ changes at least once on $(0, \infty)$ (in fact, exactly once, see [6, Proposition 2.1]). As a 
consequence, the two increments are not comparable with respect to the usual stochastic order, and hence they are not comparable either with respect to the $h r, r h$ and $l r$ orders.

\subsection{Stochastic comparisons between two EGPs}

We here provide comparison results between two different EGPs.

Proposition 6. Let $\boldsymbol{X}^{(i)}=\left(X_{t}^{(i)}\right)_{t \geq 0} \sim \Gamma\left(A_{i}(\cdot), b_{i}(\cdot)\right)$ for $i \in\{1,2\}$. Assume that:

- $A_{2}(\cdot)-A_{1}(\cdot)$ is increasing,

- $b_{1}(t) \geq b_{2}(t)$ for all $t \geq 0$.

Then, for all $t \geq 0$ and all $h \geq 0$ :

$$
X_{t+h}^{(1)}-X_{t}^{(1)} \prec_{r h} X_{t+h}^{(2)}-X_{t}^{(2)} .
$$

As a specific case, for all $t \geq 0$ :

$$
X_{t}^{(1)} \prec_{r h} X_{t}^{(2)}
$$

Proof. Let $t \geq 0$ and $h \geq 0$. Here again, we first consider the case where the functions $b_{1}(\cdot)$ and $b_{2}(\cdot)$ are piecewise constant and we proceed as in the proof of Proposition 4. It then possible to consider disjoint intervals $\left(v_{j}, v_{j+1}\right]$ for $0 \leq j \leq m$ with $v_{0}=0$ and $v_{m+1}=h$, such that $\cup_{j=0}^{m}\left(v_{j}, v_{j+1}\right]=(0, h]$ and on which both $b_{1}(t+z)$ and $b_{2}(t+z)$ are constant for all $z \in\left(v_{j}, v_{j+1}\right]$ and all $0 \leq j \leq m$. This provides:

$$
\begin{aligned}
& X_{t+h}^{(1)}-X_{t}^{(1)}=\sum_{j=0}^{m} Z_{j}^{(1)}, \\
& X_{t+h}^{(2)}-X_{t}^{(2)}=\sum_{j=0}^{m} Z_{j}^{(2)},
\end{aligned}
$$

with $Z_{j}^{(i)} \sim \Gamma_{0}\left(A_{i}\left(t+v_{j+1}\right)-A_{i}\left(t+v_{j}\right), b_{i}\left(t+v_{j+1}\right)\right)$ for $i \in\{1,2\}$.

By hypothesis, for all $j=1, \ldots, m$,

$$
A_{2}\left(t+v_{j+1}\right)-A_{1}\left(t+v_{j+1}\right) \geq A_{2}\left(t+v_{j}\right)-A_{1}\left(t+v_{j}\right),
$$

since $v_{j+1} \geq v_{j}$, which leads to,

$$
A_{2}\left(t+v_{j+1}\right)-A_{2}\left(t+v_{j}\right) \geq A_{1}\left(t+v_{j+1}\right)-A_{1}\left(t+v_{j}\right) .
$$

Moreover,

$$
b_{1}\left(t+v_{j+1}\right) \geq b_{2}\left(t+v_{j+1}\right) .
$$

From Lemma 12, it then comes:

$$
X_{t+h}^{(1)}-X_{t}^{(1)} \prec_{r h} X_{t+h}^{(2)}-X_{t}^{(2)},
$$

which achieves the proof in the case of piecewise constant scale functions. 
For general functions $b_{i}, i=1,2$, we proceed as in the proof of Proposition 1 and the result follows since $\prec_{r h}$ is preserved by convergence in distribution.

The previous proposition allows to compare an EGP and a SGP which share the same shape function, as stated in the following corollary. It is an immediate consequence and it is stated without proof.

Corollary 1. Let $\boldsymbol{Y}=\left(Y_{t}\right)_{t \geq 0} \sim \Gamma_{0}(A(t), \beta)(S G P)$ and $\boldsymbol{X}=\left(X_{t}\right)_{t \geq 0} \sim \Gamma(A(t), b(t))$ $(E G P)$. We have the following results:

- If $b(t) \leq \beta$ for all $t$ in a given interval $\left[t_{1}, t_{2}\right]$, then

$$
Y_{t+h}-Y_{t} \prec_{r h} X_{t+h}-X_{t},
$$

for all $t \in\left[t_{1}, t_{2}\left[\right.\right.$ and all $h$ such that $t+h \leq t_{2}$.

- In particular, if $b(t) \leq \beta$ for all $t \geq 0$, then

$$
Y_{t} \prec_{r h} X_{t} .
$$

- The inequalities of the two previous points are reversed if $b(t) \geq \beta$.

By considering more stringent constraints on parameter $\beta$ and function $b(\cdot)$, it is possible to give necessary and sufficient conditions for the $h r$ and $l r$ orders.

Proposition 7. Let $\boldsymbol{Y}=\left(Y_{t}\right)_{t \geq 0} \sim \Gamma_{0}(A(\cdot), \beta)(S G P)$ and $\boldsymbol{X}=\left(X_{t}\right)_{t \geq 0} \sim \Gamma(A(\cdot), b(\cdot))$ (EGP). Then:

1. Assume that there exists $b_{0}>0$ such that $b_{0} \leq b(s)$ for all $s \in[t, t+h]$ and $\int_{t}^{t+h}|\log (b(s))| a(s) d s<+\infty$. Then:

$Y_{t+h}-Y_{t} \prec_{h r} X_{t+h}-X_{t}$ is equivalent to $Y_{t+h}-Y_{t} \prec_{\text {sto }} X_{t+h}-X_{t}$ and it is true if and only if

$$
\log (\beta) \geq \frac{1}{A(t+h)-A(t)} \int_{t}^{t+h} \log (b(s)) a(s) d s .
$$

2. Assume that $\int_{t}^{t+h} b(s) a(s) d s<+\infty$. Then:

$Y_{t+h}-Y_{t} \prec_{l r} X_{t+h}-X_{t}$ is equivalent to $Y_{t+h}-Y_{t} \prec_{r h} X_{t+h}-X_{t}$ and it is true if and only if

$$
\beta \geq \frac{1}{A(t+h)-A(t)} \int_{t}^{t+h} b(s) a(s) d s .
$$

Proof. Assume first that $b(\cdot)$ is piecewise constant with $b(s)=\sum_{i=1}^{n} b_{i} \mathbf{1}_{\left(t_{i}, t_{i+1}\right]}(s)$ where $t_{1}=t, t_{n+1}=t+h$ and let $a_{i}=A\left(t_{i+1}\right)-A\left(t_{i}\right)$ for $i \in\{1, \cdots, n\}$. Then, using the notation of Lemma $4, X_{t+h}-X_{t}$ and $Y_{t+h}-Y_{t}$ are identically distributed as $S_{Z}$ and $\beta^{-1} S_{U}$, respectively. In that case, we have

$\frac{1}{A(t+h)-A(t)} \int_{t}^{t+h} \log (b(s)) a(s) d s=\frac{1}{a_{+}} \sum_{i=1}^{n} \int_{t_{i}}^{t_{i+1}} \log (b(s)) a(s) d s=\frac{1}{a_{+}} \sum_{i=1}^{n} a_{i} \log \left(b_{i}\right)$ 
and, using Lemma 4, condition (9) is equivalent to

$$
\beta \geq e^{\frac{1}{a_{+}} \sum_{i=1}^{n} a_{i} \log \left(b_{i}\right)}=\prod_{i=1}^{n} b_{i}^{\frac{a_{i}}{a_{+}}} .
$$

This proves the first point in case of a piecewise constant function $b(\cdot)$.

Now, let us consider the case of a general function $b(\cdot)$ and let $\left(b_{n}(\cdot)\right)_{n \in \mathbb{N}}$ be an increasing sequence of piecewise constant positive functions on $\mathbb{R}_{+}^{*}$, such that $b_{n}(s)$ tends to $b(s)$ when $n \rightarrow+\infty$ for all $s \in[t, t+h]$, and let $\left(X_{t}^{(n)}\right)_{t \geq 0} \sim \Gamma\left(A(\cdot), b_{n}(\cdot)\right)$.

Assume that condition (9) is true. Then, as $b_{n}(s) \leq b(s)$, condition (9) is also true when $b(s)$ is substituted by $b_{n}(s)$, and hence $Y_{t+h}-Y_{t} \prec_{h r} X_{t+h}^{(n)}-X_{t}^{(n)}$ and $Y_{t+h}-Y_{t} \prec_{s t o} X_{t+h}^{(n)}-X_{t}^{(n)}$. Letting $n$ go to infinity, we get that $Y_{t+h}-Y_{t} \prec_{h r} X_{t+h}-X_{t}$ and $Y_{t+h}-Y_{t} \prec_{\text {sto }} X_{t+h}-X_{t}$.

Conversely, assume that $Y_{t+h}-Y_{t} \prec_{s t o} X_{t+h}-X_{t}$ (or $Y_{t+h}-Y_{t} \prec_{h r} X_{t+h}-X_{t}$ ). Observe that as $\left(b_{n}(\cdot)\right)_{n \in \mathbb{N}}$ increases with $n, X_{t+h}^{(n)}-X_{t}^{(n)}$ decreases in the $r h$ ordering (due to Proposition 4.11), and hence also in the usual stochastic ordering, when $n$ increases.

Then

$$
\bar{F}_{Y_{t+h}-Y_{t}}(x) \leq \bar{F}_{X_{t+h}-X_{t}}(x)=\lim _{m \rightarrow+\infty} \bar{F}_{X_{t+h}^{(m)}-X_{t}^{(m)}}(x) \leq \bar{F}_{X_{t+h}^{(n)}-X_{t}^{(n)}}(x)
$$

for all $x \in \mathbb{R}$ and all $n \in \mathbb{N}$. This implies that $Y_{t+h}-Y_{t} \prec$ sto $X_{t+h}^{(n)}-X_{t}^{(n)}$, and hence condition (9) is true for $b_{n}(\cdot)$ :

$$
\log (\beta) \geq \frac{1}{A(t+h)-A(t)} \int_{t}^{t+h} \log \left(b_{n}(s)\right) a(s) d s
$$

for all $n \in \mathbb{N}^{*}$. As $\left|\log \left(b_{n}(s)\right)\right| \leq\left|\log \left(b_{0}\right)\right|+|\log (b(s))|$, we get by dominated convergence that

$$
\lim _{n \rightarrow+\infty} \int_{t}^{t+h} \log \left(b_{n}(s)\right) a(s) d s=\int_{t}^{t+h} \log (b(s)) a(s) d s .
$$

Then, letting $n$ go to infinity in (11) tells us that condition (9) is true for $b(\cdot)$, and the first point is true. The second point is similar and its proof is omitted.

Note that when condition (9) is not fulfilled, then $Y_{t+h}-Y_{t}$ and $X_{t+h}-X_{t}$ are not comparable with respect to the hazard rate ordering, as can be easily checked through a counter-example. The same for condition (10).

Note also that, under the conditions of Proposition 6, the results obtained for the $r h$ order are also valid for the usual stochastic order, and hence also for both increasing convex and concave orders. We now provide other conditions for the increasing convex and concave orders, which extend some results from Lemma 8 (points 4 and 6) to EGPs.

Proposition 8. Let $\boldsymbol{X}^{(i)}=\left(X_{t}^{(i)}\right)_{t \geq 0} \sim \Gamma\left(A_{i}(\cdot), b_{i}(\cdot)\right)$ for $i \in\{1,2\}$. Assume that:

- $A_{2}(\cdot)-A_{1}(\cdot)$ is decreasing, 
- $\frac{A_{2}(\cdot)}{b_{2}(\cdot)}-\frac{A_{1}(\cdot)}{b_{1}(\cdot)}$ is increasing,

- $b_{1}(\cdot)$ is decreasing and $b_{2}(\cdot)$ is increasing.

Then, for all $t \geq 0$ and all $h \geq 0$ :

$$
X_{t+h}^{(1)}-X_{t}^{(1)} \prec_{i c x} X_{t+h}^{(2)}-X_{t}^{(2)} .
$$

As a specific case, for all $t \geq 0$ :

$$
X_{t}^{(1)} \prec_{i c x} X_{t}^{(2)}
$$

Proof. Let $t \geq 0$ and $h \geq 0$. Following the proof of Proposition 6, we have:

$$
\begin{aligned}
& X_{t+h}^{(1)}-X_{t}^{(1)}=\sum_{j=0}^{m} Z_{j}^{(1)}, \\
& X_{t+h}^{(2)}-X_{t}^{(2)}=\sum_{j=0}^{m} Z_{j}^{(2)},
\end{aligned}
$$

with $Z_{j}^{(z)} \sim \Gamma_{0}\left(A_{z}\left(t+v_{j+1}\right)-A_{z}\left(t+v_{j}\right), b_{z}\left(t+v_{j+1}\right)\right)$ for $z \in\{1,2\}$.

By hypothesis, for all $j=1, \ldots, m$,

$$
A_{2}\left(t+v_{j+1}\right)-A_{1}\left(t+v_{j+1}\right) \leq A_{2}\left(t+v_{j}\right)-A_{1}\left(t+v_{j}\right),
$$

since $v_{j+1} \geq v_{j}$, which leads to,

$$
A_{2}\left(t+v_{j+1}\right)-A_{2}\left(t+v_{j}\right) \leq A_{1}\left(t+v_{j+1}\right)-A_{1}\left(t+v_{j}\right) .
$$

Moreover,

$$
\frac{A_{2}\left(t+v_{j+1}\right)}{b_{2}\left(t+v_{j+1}\right)}-\frac{A_{1}\left(t+v_{j+1}\right)}{b_{1}\left(t+v_{j+1}\right)} \geq \frac{A_{2}\left(t+v_{j}\right)}{b_{2}\left(t+v_{j}\right)}-\frac{A_{1}\left(t+v_{j}\right)}{b_{1}\left(t+v_{j}\right)} .
$$

This implies that

$$
\begin{aligned}
\frac{A_{2}\left(t+v_{j+1}\right)-A_{2}\left(t+v_{j}\right)}{b_{2}\left(t+v_{j+1}\right)} & \geq \frac{A_{2}\left(t+v_{j+1}\right)}{b_{2}\left(t+v_{j+1}\right)}-\frac{A_{2}\left(t+v_{j}\right)}{b_{2}\left(t+v_{j}\right)} \\
& \geq \frac{A_{1}\left(t+v_{j+1}\right)}{b_{1}\left(t+v_{j+1}\right)}-\frac{A_{1}\left(t+v_{j}\right)}{b_{1}\left(t+v_{j}\right)} \\
& \geq \frac{A_{1}\left(t+v_{j+1}\right)-A_{1}\left(t+v_{j}\right)}{b_{1}\left(t+v_{j+1}\right)},
\end{aligned}
$$

since $b_{1}(\cdot)$ is decreasing and $b_{2}(\cdot)$ is increasing.

From Lemma 8 , we then have for all $j=1, \ldots, m$ :

$$
Z_{j}^{(1)} \prec_{i c x} Z_{j}^{(2)} .
$$


As the increasing convex order is stable under convolution [21, Theorem 4.A.8], then

$$
X_{t+h}^{(1)}-X_{t}^{(1)} \prec_{i c x} X_{t+h}^{(2)}-X_{t}^{(2)} .
$$

We now consider a general function $b: \mathbb{R}_{+}^{*} \rightarrow \mathbb{R}_{+}^{*}$. Then, there exists two increasing sequences $\left(\beta^{(i, n)}(\cdot)\right)_{n \in \mathbb{N}}$ of piecewise constant functions of the shape $(7)$ that converges to $1 / b_{i}$ with $i \in\{1,2\}$. Let $\left(X_{t}^{(i, n)}\right)_{t \geq 0} \sim \Gamma\left(A_{i}(\cdot), b^{(i, n)}(\cdot)\right)$ with $b^{(i, n)}(t)=1 / \beta^{(i, n)}(t)$.

We have:

$$
\lim _{n \rightarrow+\infty} \mathcal{L}_{X_{t+h}^{(i, n)}-X_{t}^{(i, n)}}(\lambda)=\mathcal{L}_{X_{t+h}^{(i)}-X_{t}^{(i)}}(\lambda) \text { for all } \lambda>0
$$

by the monotone convergence theorem so that

$$
X_{t+h}^{(i, n)}-X_{t}^{(i, n)} \stackrel{\mathcal{D}}{\rightarrow} X_{t+h}^{(i)}-X_{t}^{(i)} \text { when } n \rightarrow+\infty
$$

for $i \in\{1,2\}$.

Now, we show that the previous convergence result also holds in the $L_{1}$ sense.

First note that

$$
\mathbb{E}\left[\left|\left(X_{t+h}^{(i, n)}-X_{t}^{(i, n)}\right)-\left(X_{t+h}^{(i)}-X_{t}^{(i)}\right)\right|\right] \leq \mathbb{E}\left[\left|X_{t+h}^{(i, n)}-X_{t+h}^{(i)}\right|\right]+\mathbb{E}\left[\left|X_{t}^{(i, n)}-X_{t}^{(i)}\right|\right]
$$

with

$$
\mathbb{E}\left[\left|X_{t}^{(i, n)}-X_{t}^{(i)}\right|\right] \leq \mathbb{E}\left[\int_{(0, t]}\left|\beta^{(i, n)}(s)-\frac{1}{b_{i}(s)}\right| d Y(s)\right] \underset{n \rightarrow+\infty}{\rightarrow} 0
$$

by dominated convergence, because $\left|\beta^{(i, n)}(s)-1 / b_{i}(s)\right| \leq 2 / b_{i}(s)$ and

$$
\mathbb{E}\left[\int_{(0, t]} \frac{1}{b_{i}(s)} d Y(s)\right]=\mathbb{E}\left(X_{t}^{(i)}\right)<+\infty,
$$

see Subsection 4.1.

The same reasoning shows that

$$
\mathbb{E}\left[\left|X_{t+h}^{(i, n)}-X_{t+h}^{(i)}\right|\right] \rightarrow 0,
$$

which allows to conclude from (12) that

$$
X_{t+h}^{(i, n)}-X_{t}^{(i, n)} \stackrel{L^{1}}{\rightarrow} X_{t+h}^{(i)}-X_{t}^{(i)} \text { when } n \rightarrow+\infty .
$$

Finally, the result is easily derived from [21, Theorem 4.A.8] and from the piecewise constant case.

Example 3. One can check that the assumptions of Proposition 8 are satisfied for $A_{1}(t)=\log (2 t+1), A_{2}(t)=\log (t+1), b_{1}(t)=1 /(t+1)+2$ and $b_{2}(t)=1-1 /(t+2)$. Note that this example does not fulfill the conditions of Proposition 6 , and hence Proposition 8 provides new results when compared to Proposition 6 . 
We now provide similar results for the increasing concave order. The proof follows the same steps as in Proposition 8 and it is omitted.

Proposition 9. Let $\boldsymbol{X}^{(i)}=\left(X_{t}^{(i)}\right)_{t \geq 0} \sim \Gamma\left(A_{i}(\cdot), b_{i}(\cdot)\right)$ for $i \in\{1,2\}$. Assume that:

- $b_{1}(t) \leq b_{2}(t)$ for all $t \geq 0$,

- $b_{1}(\cdot)$ is decreasing and $b_{2}(\cdot)$ is increasing,

- $\frac{A_{2}(\cdot)}{b_{2}(\cdot)}-\frac{A_{1}(\cdot)}{b_{1}(\cdot)}$ is increasing (and hence $A_{2}(\cdot)-A_{1}(\cdot)$ is also increasing).

Then, for all $t \geq 0$ and all $h \geq 0$ :

$$
X_{t+h}^{(1)}-X_{t}^{(1)} \prec_{i c v} X_{t+h}^{(2)}-X_{t}^{(2)} .
$$

As a specific case, for all $t \geq 0$ :

$$
X_{t}^{(1)} \prec_{i c v} X_{t}^{(2)} .
$$

Example 4. One can check that the assumptions of Proposition 9 are satisfied for $A_{1}(t)=\log \left(\frac{t}{2}+1\right), A_{2}(t)=\exp (t)-1, b_{1}(t)=\frac{1}{t+1}+\frac{1}{2}$ and $b_{2}(t)=2-\frac{1}{t+2}$. Here again, this example does not fulfill the conditions of Proposition 6, and Proposition 9 provides new results when compared to Proposition 6.

\subsection{Aging properties of threshold crossing times}

We finally come to aging properties of threshold crossing times. We begin with a first result when the threshold is an IFR random variable.

Proposition 10. Let $L$ be an IFR non negative random variable independent of an $E G P \boldsymbol{X}$ with $X_{t} \sim \Gamma(A(t), b(t))$. Let

$$
\sigma_{L}=\inf \left\{t \geq 0: X_{t} \geq L\right\} .
$$

Assume that

- $A(\cdot)$ is convex,

- $b(\cdot)$ is decreasing.

Then, $\sigma_{L}$ is IFR.

Proof. Let us first note that, by construction, any EGP $\boldsymbol{X}$ belongs to the class IPII (Independent Positive Increasing Increments) in the vocabulary of [18, Definition 2.7]. Also, based on Proposition $4, \boldsymbol{X}$ increases with respect to the $r h$ order. Then, the result is a direct consequence of [18, Proposition 3.1].

We derive the following corollary, considering a deterministic threshold $\ell$.

Corollary 2. Let $\ell>0$ and let $\boldsymbol{X}$ be an EGP with $X_{t} \sim \Gamma(A(t), b(t))$. Let

$$
\sigma_{\ell}=\inf \left\{t \geq 0: X_{t} \geq \ell\right\} .
$$

Assume that

- $A(\cdot)$ is convex, 
- $b(\cdot)$ is decreasing,

Then, $\sigma_{\ell}$ is IFR.

Proof. For $n \in \mathbb{N}^{*}$, let $L^{(n)} \sim \Gamma_{0}(n \ell, n)$. As $L^{(n)}$ is IFR for a sufficiently large $n$, we know from Proposition 10 that $\sigma_{L^{(n)}}$ is IFR. Also, as $\mathbb{E}\left(\left(L^{(n)}-\ell\right)^{2}\right)=\operatorname{var}\left(L^{(n)}\right)=\frac{\ell}{n} \rightarrow 0$ when $n \rightarrow+\infty$, we have $L^{(n)} \stackrel{\mathcal{D}}{\rightarrow} \ell$ when $n \rightarrow+\infty$. We now have

$$
\mathbb{P}\left(\sigma_{L^{(n)}}>t\right)=\mathbb{P}\left(X_{t} \leq L^{(n)}\right) \rightarrow \mathbb{P}\left(X_{t} \leq \ell\right)=\mathbb{P}\left(\sigma_{\ell}>t\right)
$$

for all $t$ so that $\sigma_{L^{(n)}} \stackrel{\mathcal{D}}{\rightarrow} \sigma_{\ell}$. As IFR is stable through convergence in distribution, we derive that $\sigma_{\ell}$ is IFR.

We next consider the DRHR property. Recall that for a SGP, the concavity of $A(\cdot)$ is a sufficient condition to ensure that $\sigma_{\ell}$ is DRHR. This is not the case for an EGP, neither when $b(\cdot)$ is decreasing nor when it is increasing, whatever the convexity/concavity of the shape function is, as show the two following examples.

Example 5. Let $A(t)=t$. We consider a decreasing scale function $b(t)=2 \mathbb{1}_{[0,0.25)}(t)+$ $1 \mathbb{1}_{[0.25, \infty)}(t)$. As can be seen in Figure 3 (left), the function $\log \left(F_{\sigma_{2}}\right)$ is not concave and hence $\sigma_{2}$ is not DRHR. In Figure 3 (right), we can notice that the function $\log \left(\bar{F}_{\sigma_{2}}\right)$ is concave so that $\sigma_{2}$ is IFR. This is coherent with the result of the Corollary 2.
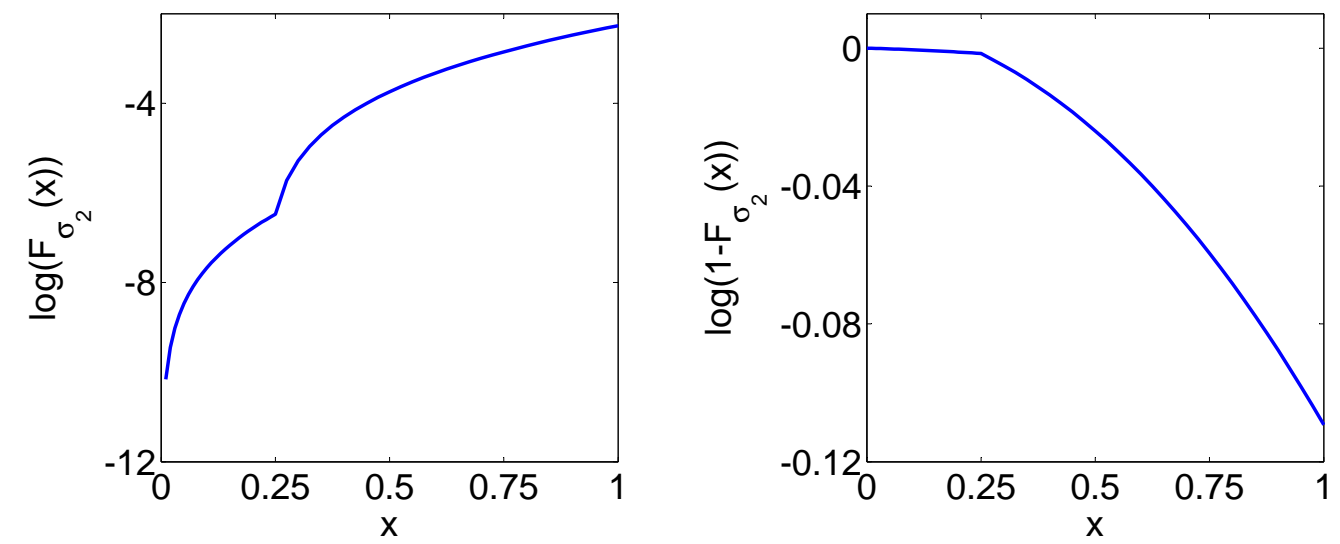

Figure 3: Plots of $\log \left(F_{\sigma_{2}}\right)$ and $\log \left(\bar{F}_{\sigma_{2}}\right)$ for $A(t)=t$ and $b(t)=2 \mathbb{1}_{[0,0.25)}(t)+$ $1 \mathbb{1}_{[0.25, \infty)}(t)$

Example 6. Let $A(t)=t$. We now consider an increasing scale function $b(t)=$ $0.1 \mathbb{1}_{[0,0.25)}(t)+10 \mathbb{1}_{[0.25, \infty)}(t)$ As can be seen in Figure 4 (left), $\log \left(F_{\sigma_{2}}\right)$ is not concave and $\sigma_{2}$ is hence not DRHR. In addition, we can observe in Figure 4 (right) that the function $\log \left(\bar{F}_{\sigma_{2}}\right)$ is not concave and hence $\sigma_{2}$ is not IFR.

\section{Recap and perspectives}

Stochastic comparisons and aging properties have been found, considering an EGP 

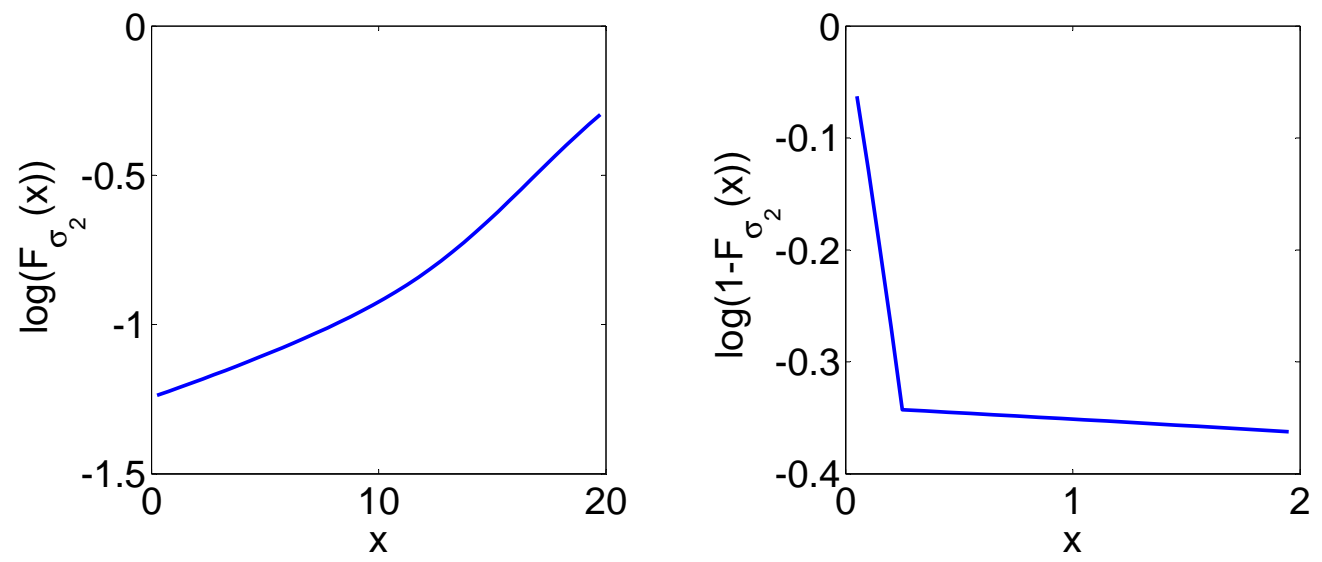

Figure 4: Plots of $\log \left(F_{\sigma_{2}}\right)$ and $\log \left(\bar{F}_{\sigma_{2}}\right)$ for $A(t)=t$ and $b(t)=0.1 \mathbb{1}_{[0,0.25)}(t)+$ $10 \mathbb{1}_{[0.25, \infty)}(t)$

as a model for cumulative deterioration in reliability theory. Note that the results can naturally be used in other contexts, such as finance or risk theory for instance.

Most of the results of the paper are summed up in Table 1, where previous results for SGPs are recalled, for comparison purpose with their extended version (EGPs). Note that the comparison results between a SGP and an EGP from Corollary 1 and Proposition 7 do not appear in this table, for a better readability.

As can be seen in Table 1, the results previously obtained for SGPs and dealing with the DFR/DRHR property of an increment remain true. Those dealing with $i c x / i c v$ comparison results and the IFR property of a crossing time have a natural extension, adding a monotonicity assumption on the scale function(s) of the $\operatorname{EGP}(\mathrm{s})$. However, we can notice that the $l r$ (and $h r$ ) results for SGPs do not remain valid considering EGPs, and only results with the weaker $r h$ order have been obtained (with counter-examples showing that the results cannot be extended to the $l r$ order in most cases). Also, we remind to the reader that we have not been able to conclude whether $X_{s} \prec_{l r} X_{t}$ for $s<t$ could be valid assuming the shape function to be convex or concave, and the scale function to be increasing (see Remark 2). This (challenging) question hence remains open.

Clearly, there remain many other stochastic orders (and aging properties) to be investigated in the context of an EGP, such as the stochastic precedence order [3], for which one might hope to obtain results under weaker conditions than for the usual stochastic order studied in this paper, or for more technical stochastic orders such as (harmonic) mean residual life order, dispersive order, excess wealth order, convex order, to quote only a few. (We refer to the seminal books quoted in the introduction, where the interested reader can find further stochastic ordering and aging notions, for which the degree of importance may vary according to the application context). There hence remain many investigation needed for a better understanding of the possible stochastic behavior of an EGP, even restricting the study to the domain of stochastic comparisons and aging properties. 
TABle 1: Aging properties and stochastic ordering for SGPs (second column) and EGPs (third column)

\begin{tabular}{|c|c|c|}
\hline \multicolumn{3}{|c|}{ Aging properties of $X_{t+h}-X_{t}($ with $t, h \geq 0)$} \\
\hline & $\boldsymbol{X} \sim \Gamma_{0}(A(\cdot), b) \mathbf{S G P}$ & $\boldsymbol{X} \sim \Gamma(\bar{A}(\cdot), b(\cdot)) \mathbf{E G P}$ \\
\hline$X_{t+h}-X_{t}$ IFR & true if $A(t+h)-A(t) \geq 1$ & $\begin{array}{l}\text { not true (even if } A(\cdot) \text { is con- } \\
\text { vex/concave and } b \text { is monotonous) }\end{array}$ \\
\hline$X_{t+h}-X_{t}$ DFR & true if $A(t+h)-A(t) \leq 1$ & true if $A(t+h)-A(t) \leq 1$ \\
\hline$X_{t+h}-X_{t}$ DRHR & always true & always true \\
\hline \multicolumn{3}{|c|}{ Monotonicity and comparison of increments (with $0 \leq s \leq t$ ) and $h \geq 0$} \\
\hline & $\dot{\boldsymbol{X}} \sim \Gamma_{0}(A(\cdot), b) \mathbf{S G P}$ & $\boldsymbol{X} \sim \Gamma(\bar{A}(\cdot), \bar{b}(\cdot)) \mathbf{E G P}$ \\
\hline$X_{s} \prec X_{t}$ & lr order always true & $\begin{array}{l}\text { - } r h \text { order always true } \\
\text { - } h r(\text { and } l r) \text { order not true when } b(\cdot) \\
\text { increases }\end{array}$ \\
\hline $\begin{array}{l}X_{s+h}-X_{s} \prec \\
X_{t+h}-X_{t}\end{array}$ & lr order true if $A(\cdot)$ is convex & $\begin{array}{l}r h \text { order if } A(\cdot) \text { is convex and } b(\cdot) \\
\text { decreases }\end{array}$ \\
\hline $\begin{array}{l}X_{s+h}-X_{s} \succ \\
X_{t+h}-X_{t}\end{array}$ & lr order true if $A(\cdot)$ is concave & $\begin{array}{l}r h \text { order if } A(\cdot) \text { is concave and } b(\cdot) \\
\text { increases }\end{array}$ \\
\hline \multicolumn{3}{|c|}{ Comparisons between two processes } \\
\hline & $\begin{array}{l}\boldsymbol{X}^{(1)} \sim \Gamma_{0}\left(A_{1}(\cdot), b_{1}\right) \text { SGP } \\
\boldsymbol{X}^{(2)} \sim \Gamma_{0}\left(A_{2}(\cdot), b_{2}\right) \text { SGP }\end{array}$ & $\begin{array}{l}\boldsymbol{X}^{(1)} \sim \Gamma\left(A_{1}(\cdot), b_{1}(\cdot)\right) \text { EGP } \\
\boldsymbol{X}^{(2)} \sim \Gamma\left(A_{2}(\cdot), b_{2}(\cdot)\right) \text { EGP }\end{array}$ \\
\hline $\begin{array}{l}X_{t+h}^{(1)}-X_{t}^{(1)} \prec \\
X_{t+h}^{(2)}-X_{t}^{(2)}\end{array}$ & $\begin{array}{l}\text { - } l r \text { order if } A_{2}(\cdot)-A_{1}(\cdot) \text { is increasing } \\
\text { and } b_{1} \geq b_{2} \\
\text { - } i c x \text { order if } A_{2}(\cdot)-A_{1}(\cdot) \text { is decreas- } \\
\text { ing and } \frac{A_{2}(\cdot)}{b_{2}}-\frac{A_{1}(\cdot)}{b_{1}} \text { is increasing } \\
\text { - } i c v \text { order if } b_{1} \leq b_{2} \text { and } \frac{A_{2}(\cdot)}{b_{2}}-\frac{A_{1}(\cdot)}{b_{1}} \\
\text { is increasing }\end{array}$ & $\begin{array}{l}\text { - } r h \text { order if } A_{2}(\cdot)-A_{1}(\cdot) \text { is increasing } \\
\text { and } b_{1}(t) \geq b_{2}(t) \\
\text { - } i c x \text { order if } A_{2}(\cdot)-A_{1}(\cdot) \text { is de- } \\
\text { creasing, } \frac{A_{2}(\cdot)}{b_{2}}-\frac{A_{1}(\cdot)}{b_{1}} \text { increasing, } b_{1}(\cdot) \\
\text { decreasing and } b_{2}(\cdot) \text { increasing } \\
\text { - } i c v \text { order } \frac{A_{2}(\cdot)}{b_{2}(\cdot)}-\frac{A_{1}(\cdot)}{b_{1}(\cdot)} \text { is increasing, } \\
b_{1}(\cdot) \text { decreasing and } b_{2}(\cdot) \text { increasing } \\
\text { and } b_{1}(t) \leq b_{2}(t)\end{array}$ \\
\hline \multicolumn{3}{|c|}{ Aging properties of a crossing time $\sigma_{\ell}$} \\
\hline & $\boldsymbol{X} \sim \Gamma_{0}(A(\cdot), b)$ & $\boldsymbol{X} \sim \Gamma(A(\cdot), b(\cdot))$ \\
\hline $\begin{array}{l}\sigma_{\ell} \text { IFR } \\
\sigma_{\ell} \text { DRHR }\end{array}$ & $\begin{array}{l}\text { true if } A(\cdot) \text { is convex } \\
\text { true if } A(\cdot) \text { is concave }\end{array}$ & $\begin{array}{l}\text { true if } A(\cdot) \text { is convex and } b(\cdot) \text { is } \\
\text { decreasing } \\
\text { not true even if } A(\cdot) \text { is concave and } \\
b(\cdot) \text { increases or decreases }\end{array}$ \\
\hline
\end{tabular}

\section{References}

[1] Al Masry, Z., Mercier, S. And Verdier, G. (2017). Approximate simulation techniques and distribution of an extended gamma process. Methodol. Comput. Appl. Probab., 19, 213-235.

[2] Al Masry, Z., Mercier, S. and Verdier, G. (2018). Generalized method of moments for an extended gamma process. Commun. Stat.-Theor. M., 47:15, 3687-3714.

[3] Boland, P.J., Singh, H., Cukic, B. (2004). The stochastic precedence ordering with applications in sampling and testing. Journal of Applied Probability, 41, 73--82.

[4] ÇInlar (1980). On a generalization of gamma processes. J. Appl. Probab., 17, 467-480.

[5] Denuit, M., Dhaene, J., Goovaerts, M. and Kaas, R. (2006). Actuarial theory for dependent risks: measures, orders and models, John Wiley \& Sons. 
[6] Diaconis, P. and Perlman, M. D. (1990). Bounds for tail probabilities of weighted sums of independent gamma random variables. Lecture Notes-Monograph Series, pages 147-166.

[7] Doksum, K. A. And Hoyland, A. (1992). Models for variable-stress accelerated life testing experiment based on wiener processes and the inverse gaussian distribution. Technometrics, 34, $74-82$.

[8] Doksum, K. A. And Normand, S.-L. T. (1995). Gaussian models for degradation processes part i: Methods for the analysis of biomarker data. Lifetime Data Anal., 1, 131-144.

[9] Dykstra, R. L. And Laud, P. (1981). A bayesian nonparametric approach to reliability. Ann. Stat., 9:2, 356-367.

[10] Guida, M. And Pulcini, G. (2013). The inverse gamma process: A family of continuous stochastic models for describing state-dependent deterioration phenomena. Reliab. Eng. Syst. Saf., 120:0, $72-79$.

[11] Guida, M., Postiglione, F. and Pulcini, G. (2012). A time-discrete extended gamma process for time-dependent degradation phenomena. Reliab. Eng. Syst. Saf., 105, 73-79.

[12] Kahle, W., Mercier, S. and Paroissin, C. (2016). Degradation Processes in Reliability, WileyISTE.

[13] Kochar, S. (2012). Stochastic comparisons of order statistics and spacings: a review. ISRN Probability and Statistics.

[14] LaI, C. D. And XIE, M. (2006). Stochastic ageing and dependence for reliability, Springer, New York.

[15] Marshall, A. W. and Olkin, I. (2007). Life Distributions: Structure of Nonparametric, Semiparametric, and Parametric Families, Springer Series in Statistics. Springer.

[16] Müller, A. And Stoyan, D. (2002). Comparison Methods for Stochastic Models and Risks, John Wiley \& Sons Ltd., Chichester.

[17] Rolski, T., Schmidli, H., Schmidt, V.r and Teugels, J. (1999). Stochastic processes for insurance and finance, Wiley Series in Probability and Statistics, John Wiley \& Sons Ltd., Chichester.

[18] Sangüesa, C., Badía, F. G. and Cha, J. H. (2014). Preservation of ageing classes in deterioration models with independent increments. J. Inequal. Appl., 1-16

[19] Sato, K. (1999). Lévy processes and infinitely divisible distributions, volume 68 of Cambridge Studies in Advanced Mathematics, Cambridge University Press, Cambridge.

[20] Sengupta, D. and Nanda, A. K. (1999). Log-concave and concave distributions in reliability. Nav. Res. Log., 46:4, 419-433.

[21] Shaked, M. and Shanthikumar, J. G. (2007). Stochastic Orders, Springer, New York.

[22] Stoyan, D. (1983). Comparison methods for queues and other stochastic models, Wiley Series in Probability and Mathematical Statistics: Applied Probability and Statistics. John Wiley \& Sons Ltd., Chichester.

[23] Sumita, U. and Shanthikumar, J.G. (1985). A class of correlated cumulative shock models. Adv. Appl. Probab., 17:2, 347-366.

[24] VAn Noortwijk, J. M. (2009). A survey of the application of gamma processes in maintenance. Reliab. Eng. Syst. Safe., 94, 2-21.

[25] Ye, Z. S. And Chen, N. (2014). The inverse gaussian process as a degradation model. Technometrics, 56:3, 302-311. 
[26] YU, Y. (2009). Stochastic ordering of exponential family distributions and their mixturesxk. J. Appl. Probab., 46:1, 244-254.

[27] ZhнO, P. ( 2011). Some new results on convolutions of heterogeneous gamma random variables. J. Multivariate Anal., 102:5, 958-976. 